\title{
GLUCOSE-INSULIN-POTASSIUM SOLUTIONS ENHANCE RECOVERY AFTER URGENT CORONARY ARTERY BYPASS GRAFTING
}

\author{
Harold L. Lazar, MD ${ }^{\mathrm{a}}$ \\ George Philippides, $\mathrm{MD}^{\mathrm{b}}$ \\ Carmel Fitzgerald, $\mathrm{RN}^{\mathrm{c}}$ \\ Diane Lancaster, $\mathrm{PhD}^{\mathrm{c}}$ \\ Richard J. Shemin, $\mathrm{MD}^{\mathrm{a}}$ \\ Carl Apstein, $\mathrm{MD}^{\mathrm{b}}$
}

\begin{abstract}
Objective: This prospective, randomized, clinical study was undertaken to determine whether glucose-insulin-potassium solutions would benefit patients undergoing coronary artery bypass grafiting because of unstable angina. Methods: The study group consisted of 30 patients with unstable angina who required coronary artery bypass grafting. In 15 patients, glucose-insulin-potassium solution $\left(30 \%\right.$ dextrose in water; $\mathrm{K}^{+}, 80 \mathrm{mEq} / \mathrm{L}$; regular insulin, 50 units) was given intravenously at $1 \mathrm{ml} / \mathrm{kg}$ per hour after induction of anesthesia and administration continued for 12 hours after aortic unclamping. Fifteen patients in a separate group received 5\% dextrose in water intravenously at $50 \mathrm{ml} / \mathrm{hr}$. Results: Patients treated with glucose-insulin-potassium solution had higher cardiac indices $(2.8 \pm 0.1 \mathrm{vs}$ $2.0 \pm 1 \mathrm{~L} / \mathrm{min}$ per square meter; $p<0.001)$, lower inotrope scores $(0.06 \pm$ 0.01 vs $0.46 \pm 0.19 ; p=0.041)$, and less weight gain $(6.4 \pm 9$ vs $11.6 \pm 1.1$ pounds; $p<0.001)$ and had shorter times of ventilator support $(8.3 \pm 0.6$ vs $14.2 \pm 0.2$ hours; $p=0.003$ ). They had a significantly lower incidence of atrial fibrillation $(13.3 \%$ vs $53.3 \% ; p=0.020)$ and had shorter stays in the intensive care unit $(14.8 \pm 1.3$ vs $31.6 \pm 5.2$ hours; $p=0.002)$ and in the hospital (6.0 \pm 0.4 vs $8.0 \pm 0.7$ days; $p=0.010)$. Conclusions: We conclude that glucose-insulin-potassium therapy enhances myocardial performance and results in faster recovery from urgent coronary artery bypass grafting. (J Thorac Cardiovasc Surg 1997;113:354-62)
\end{abstract}

A lthough the operative morbidity and mortality rates for elective coronary artery bypass grafting (CABG) have remained stable, urgent and emergency $\mathrm{CABG}$ for unstable angina continues to result in higher rates of mortality and morbidity. ${ }^{1}$ The increased ischemic myocardial damage seen in these patients results in decreased ventricular function, increased fluid retention, prolonged mechanical ventilation and pulmonary dysfunction, and a higher

From the Departments of Cardiothoracic Surgery, ${ }^{a}$ Cardiology, ${ }^{b}$ and Nursing, ${ }^{c}$ The Boston University Medical Center, Boston, Mass.

Read at the Twenty-second Annual Meeting of The Western Thoracic Surgical Association, Maui, Hawaii, June 26-29, 1996.

Received for publication July 3, 1996; revisions requested August 26, 1996; revisions received Sept. 23, 1996; accepted for publication Sept. 24, 1996.

Address for reprints: Harold L. Lazar, MD, Department of Cardiothoracic Surgery, Suite B404, The Boston University Medical Center Hospital, 88 E. Newton St., Boston, MA 02118.

Copyright (C) 1997 by Mosby-Year Book, Inc.

$0022-5223 / 97 \$ 5.00+0 \quad \mathbf{1 2 / 6 / 7 8 1 7 5}$ incidence of arrhythmia, all of which contribute to longer hospitalizations and increased medical costs.

Substrate enhancement with glucose is an intervention that may limit myocardial necrosis during urgent surgical revascularization. Although free fatty acids are the main energy source for the nonischemic myocardium, glucose is a more favorable energy source during periods of ischemia and reperfusion and in chronically dysfunctional but viable myocardium., ${ }^{2,3}$ In 1965, Sodi-Pollaris and colleagues ${ }^{4}$ used glucose-insulin-potassium (GIK) solutions in patients with acutely infarcting myocardium and found that they limited electrocardiographic changes. Subsequent studies that used GIK solutions during periods of ischemia in experimental preparations and in clinical studies involving patients with acute myocardial infarctions and after cardiac surgical procedures demonstrated that GIK solutions enhanced myocardial performance..$^{5-12}$

These favorable results prompted us to perform an experimental study in pigs that simulated the conditions of urgent surgical revascularization to determine whether GIK solutions would decrease myocardial damage. ${ }^{13}$ Animals underwent 90 min- 
utes of coronary occlusion followed by 30 minutes of cardioplegic arrest and 180 minutes of reperfusion. Hearts treated with GIK solution had a significant decrease in the incidence of ventricular arrhythmia, less myocardial acidosis, better preservation of wall motion, and the lowest areas of tissue necrosis. The best protection from ischemic damage was obtained when GIK solution was given before and during the period of coronary occlusion, as well as during reperfusion. These experimental results prompted us to investigate the role of GIK solution during CABG in patients with unstable coronary syndromes. We sought to determine whether GIK solution would not only enhance myocardial performance but also result in a faster recovery from CABG operation thereby reducing both intensive care unit (ICU) and total hospital stay.

\section{Methods}

Approval to use GIK solution in human subjects was obtained from the Boston University Medical Center Institutional Review Board (protocol E3270/A65). An informed consent was obtained from each patient enrolled in the study.

Eligibility and exclusion criteria. Eligibility criteria for patients in this study included all. patients with unstable angina. Unstable angina was defined as the presence of angina at rest necessitating intravenous nitroglycerin, intravenous heparin, or intraaortic balloon pump (IABP) therapy. Patients with diabetes mellitus, chronic renal failure (creatinine level $\geq 2.0 \mathrm{mg} / \mathrm{ml}$ ), acute renal failure (urine output $<20 \mathrm{ml} / \mathrm{hr} \times 3$ ), hyperkalemia (K level $\geq 5.5$ $\mathrm{mEq} / \mathrm{L}$ ), or hepatic insufficiency (total bilirubin level $\geq 2.5$ $\mathrm{mg} / \mathrm{ml}$; levels of aspartate aminotransferase and alanine aminotransferase $>100 \mathrm{IU}$ ) were excluded from the study. In addition, patients who required procedures in combination with CABG (valve repair/replacement, aneurysm repair) were excluded. Patients who could not tolerate the GIK infusion because of hyperglycemia (glucose level $\geq 325 \mathrm{mg} / \mathrm{L})$ or hyperkalemia $\left(\mathrm{K}^{+}\right.$level $\left.\geq 5.5 \mathrm{mEq} / \mathrm{L}\right)$ were also excluded.

Study protocol. Patients who met the eligibility criteria for this study were randomized to a GIK or no-GIK group on the basis of the last digit of their hospital identification number. All patients underwent placement of a central intravenous catheter in the holding area before induction of anesthesia at which time a Swan-Ganz thermodilution catheter (Baxter Healthcare Corp., Edwards Division, Irvine, Calif.) was inserted along with a radial artery catheter. After the central line and Swan-Ganz catheter were inserted, those patients randomized to the GIK arm of the study received an infusion at $1 \mathrm{ml} / \mathrm{kg}$ per hour of GIK solution consisting of a liter bag of $30 \%$ dextrose in water with $80 \mathrm{mEq}$ of $\mathrm{KCl}$ and 50 units regular insulin. The infusion of the GIK solution continued through the induction period and the time before cardiopulmonary bypass. After cardiopulmonary bypass was initiated, the infusion of the GIK solution was stopped. The infusion was restarted immediately after aortic unclamping and continued for 12 hours. The no-GIK group received 5\% dextrose in water infused at $50 \mathrm{ml} / \mathrm{hr}$.

Operative techniques. Standard operative and anesthetic techniques were used in all patients. Anesthesia was induced with fentanyl ( 25 to $50 \mu / \mathrm{kg}$ ) and pancuronium bromide (Pavulon, $0.1 \mathrm{mg} / \mathrm{kg}$ ). Patients were supported by cardiopulmonary bypass by means of an arterial cannula in the ascending aorta and a single venous cannula in the right atrium. All vessels that had stenoses of $50 \%$ or more were bypassed. At least one internal thoracic artery was used in each patient. Proximal anastomoses were done during cardiopulmonary bypass with the heart beating followed by the construction of all distal anastomoses during one period of ischemic arrest. Myocardial protection was achieved by multidose infusions of antegrade cold blood cardioplegic solution $\left(4^{\circ} \mathrm{C} ; \mathrm{K}, 28 \mathrm{mEq} / \mathrm{L} ; \mathrm{pH}\right.$, 7.6 ; hematocrit, $20 \%)$ supplemented with systemic $\left(32^{\circ}\right.$ to $34^{\circ} \mathrm{C}$ ) and topical (cold saline solution lavage at $4^{\circ} \mathrm{C}$ ) hypothermia.

Parameters measured and data acquisition. Serum glucose and potassium levels were measured before infusion of GIK solution, before the initiation of cardiopulmonary bypass, during cardiopulmonary bypass, and every 4 hours after aortic unclamping for 18 hours. Electrocardiograms were obtained before operation, immediately after operation, and on postoperative days 1, 2, 5, and 7 . The myocardial fraction of creatinine kinase and lactate dehydrogenase isoenzyme level were measured immediately after operation and at 6 and 24 hours after operation. A perioperative myocardial infarction was diagnosed either by the appearance of new changes on the electrocardiogram ( $Q$ waves, ST segment elevation $\geq 1 \mathrm{~mm}$, loss of $R$ wave in precordial leads) or by the elevation of creatine kinase MB levels to greater than $50 \mathrm{IU}$ in the immediate 24-hour period after operation. Cardiac index was derived from thermodilution cardiac output measurements made hourly for 18 hours after operation.

Inotropic agents were used to maintain a cardiac index of $2.0 \mathrm{~L} / \mathrm{min}$ per square meter or higher and a systolic blood pressure of $90 \mathrm{~mm} \mathrm{Hg}$ or higher after afterload, preload, and heart rate were maximized. In addition to reporting the actual incidence of use of inotropic agents, an inotropic score was also used to quantify the number of inotropes used, the dosage, and length of administration. The score ranged from 0 to 5 where 0 indicated no inotropes or dopamine $2 \mu \mathrm{g} / \mathrm{kg}$ per minute or less, 1 indicated inotropic support $2 \mu \mathrm{g} / \mathrm{kg}$ per minute or greater for 24 hours, 2 indicated use of two inotropes, 3 indicated use of epinephrine, 4 indicated use of three inotropes, and 5 indicated inotropic support for 24 hours or more.

Atrial pacing was instituted if the heart rate was 80 beats/min or less with a cardiac index of $2.0 \mathrm{~L} / \mathrm{min}$ per square meter or less. All patients were weighed the evening before operation and at 5 AM the day after operation to determine postoperative weight gain in pounds. The time spent on ventilator support was recorded in hours from the time of admission to the ICU to the time of extubation. Standardized weaning protocols were used in all patients. Criteria for extubation included an inspiratory force of $\leq 20 \mathrm{~cm}$, a respiratory rate less than 30 breaths/min, oxygen saturation $90 \%$ or 
greater, $\mathrm{pH} 7.35$ to 7.45 , and carbon dioxide tension less than $50 \mathrm{~mm} \mathrm{Hg}$. The incidence of atrial fibrillation was recorded for all patients along with the occurrence of ventricular arrhythmias. A documented ventricular arrhythmia consisted of either ventricular tachycardia or multifocal premature ventricular contractions necessitating drug therapy. Administration of $\beta$-blockers was instituted before operation and on the first postoperative day in all patients with an ejection fraction of $40 \%$ or greater.

Length of stay in the ICU was recorded for all patients. Before transfer from the ICU, patients had to be extubated and have stable vital signs without any inotropic support. Hospital length of stay was defined as the time in the hospital from the day of the operation to the day of discharge. Criteria for discharge included normal sinus rhythm, a temperature of less than $99^{\circ} \mathrm{F}$, well-healed incisions, and the ability to walk and climb one flight of stairs with an oxygen saturation greater than $90 \%$ while breathing room air.

Statistical analyses. Data are presented as the actual number of occurrences in a group and as the mean plus or minus the standard error. Analysis by $\chi^{2}$ test was used to compare occurrences between the GIK and no-GIK groups. Nonpaired Student's $t$ tests were used to compare measured data between the groups. Data were considered significant at a $p$ value of less than 0.05 .

\section{Results}

Thirty-one patients were enrolled in the study. Sixteen patients were randomized to receive GIK solution. One patient was excluded from this group when the blood sugar value exceeded $325 \mathrm{mg} / \mathrm{L} 10$ hours into the infusion. The infusion was stopped and the blood sugar value decreased to $180 \mathrm{mg} / \mathrm{L}$. This patient had an uncomplicated postoperative course. All 15 patients randomized to the no-GIK protocol completed the study.

Patient profiles and cardiac risk factors. The mean age (GIK group: 60 years, range 50 to 80 , vs no-GIK group: 65 years, range 39 to 83 ) and ratio of male to female patients $(11 / 4$ GIK vs $10 / 5$ no-GIK group) were similar in both groups. All 30 patients received intravenous heparin treatment before CABG. A slightly higher percentage of patients in the GIK group received intravenous nitroglycerin therapy (12/15 vs $8 / 15)$ and had a prior myocardial infarction $(14 / 15$ vs $11 / 15)$. The incidence of congestive heart failure (4/15 GIK vs $3 / 15$ no-GIK group), the need for preoperative IABP support (3/15 GIK vs 4/15 no-GIK group), and the mean ejection fraction (GIK: $43 \%$, range $20 \%$ to $50 \%$, vs no-GIK: $44 \%$, range $30 \%$ to $50 \%$ ) were similar in the two groups. The majority of patients in both groups had hypertension (12/15 GIK vs $10 / 15$ no-GIK group) whereas only a small percentage had chronic obstructive pulmonary disease $(3 / 15$
GIK vs $1 / 15$ no-GIK group) or a previous cerebral vascular accident (1/15 GIK vs $2 / 15$ no-GIK group).

Operative results. There was no difference between the groups in the duration of cardiopulmonary bypass $(101.2 \pm 2.2$ minutes GIK vs $98.6 \pm 6.1$ minutes no-GIK group, $p=0.370$ ) or the crossclamp time $(43.8 \pm 2.6$ minutes $\mathrm{GIK}$ vs $41.3 \pm 2.4$ minutes no-GIK group; $p=0.240$ ). The GIK group had a slightly higher number of vessels bypassed, which was nonetheless significant $(3.46 \pm 0.16$ vs $3.00 \pm 0.09 ; p=$ $0.010 ; 95 \%$ confidence interval $[\mathrm{CI}]-0.86$ to 0.07 ).

Serum $\mathrm{K}^{+}$and glucose levels. Levels of serum $\mathrm{K}^{+}$remained constant in both the GIK and no-GIK groups during the prebypass and reperfusion periods (Fig. 1).

There was no difference in serum glucose levels before the infusion of GIK solution (108 $\pm 6 \mathrm{mg} / \mathrm{L}$ GIK vs $110 \pm 5 \mathrm{mg} / \mathrm{L}$ no-GIK group; $p=0.410$ ) (Fig. 2). Glucose levels increased significantly before bypass in the GIK group and remained elevated during the infusion of the GIK solution in the reperfusion period $(220 \pm 10 \mathrm{mg} / \mathrm{L}$ at 6 hours and $240 \pm 10 \mathrm{mg} / \mathrm{L}$ at 12 hours $)$. However, after the discontinuation of the GIK infusion, there was no difference in serum glucose level between the two groups ( $140 \pm 8 \mathrm{mg} / \mathrm{L}$ GIK vs $135 \pm 5 \mathrm{mg} / \mathrm{L}$ no-GIK group at 18 hours of reperfusion; $p=0.350$ ).

Postoperative results. There were no deaths in either group. One patient in each group had a perioperative myocardial infarction. The postoperative cardiac indices for both groups are summarized in Fig. 3. Although both groups started out with similar indices (GIK group: $2.1 \pm 0.2$ L/min per square meter vs no-GIK group: $2.1 \pm$ $0.2 \mathrm{~L} / \mathrm{min}$ per square meter; $p=0.510$ ) the patients treated with GIK solution had higher cardiac indices during reperfusion that persisted even after the GIK infusion was discontinued (GIK group: $2.8 \pm 0.1 \mathrm{~L} / \mathrm{min}$ per square meter vs no-GIK group: $2.3 \pm 0.2 \mathrm{~L} / \mathrm{min}$ per square meter; $p<0.001 ; 95 \% \mathrm{CI}-1.20$ to 0.312 ). Patients in the GIK group required less inotropic support (1/15 vs $5 / 15 ; p=0.080$ ) and had significantly lower inotropic scores $(0.06 \pm 0.01$ vs $0.46 \pm 0.19 ; p=$ $0.041 ; 95 \% \mathrm{CI} 0.31$ to 3.81 ). They also required less atrial pacing $(2 / 15$ vs $10 / 15 ; p=0.002)$. Patients treated with GIK solution gained less weight (GIK group: $6.4 \pm 0.9$ pounds vs no-GIK group: $11.6 \pm 1.1$ pounds; $p<0.001 ; 95 \%$ CI 2.08 to 8.82) and had shorter times of ventilator support $(8.3 \pm 0.6$ hours GIK vs $14.2 \pm 0.2$ hours no-GIK group; $p=0.003 ; 95 \%$ CI 1.62 to 10.23 ). 


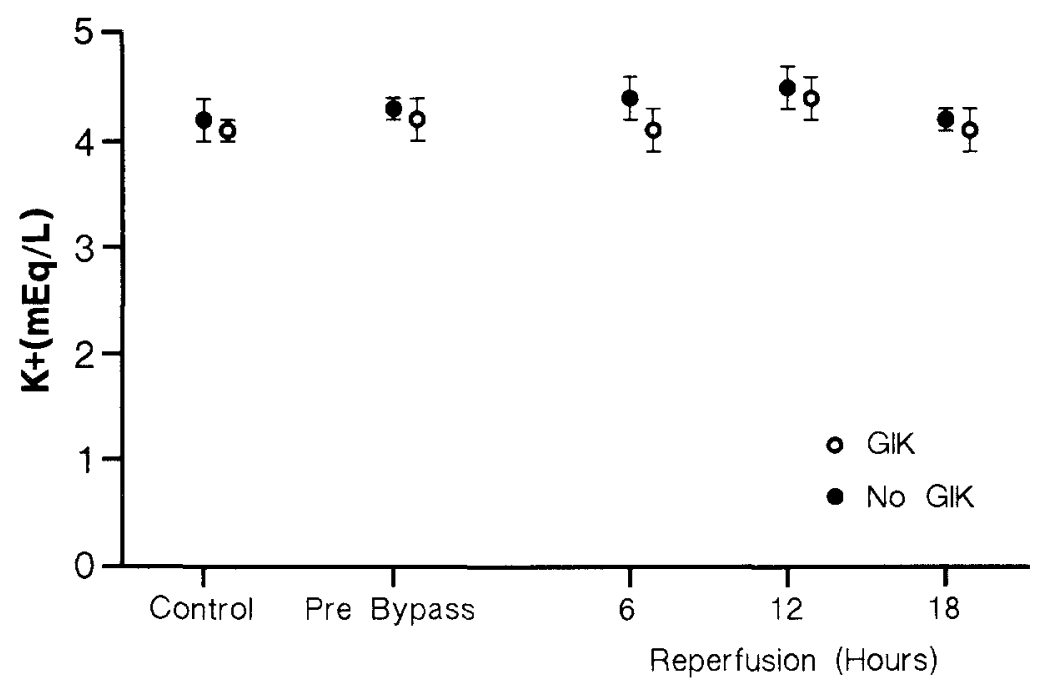

Fig. 1. Serum $\mathrm{K}^{+}$levels. All values represent the mean plus or minus the standard error. Serum $\mathrm{K}^{+}$levels do not differ between the GIK and no-GIK groups.

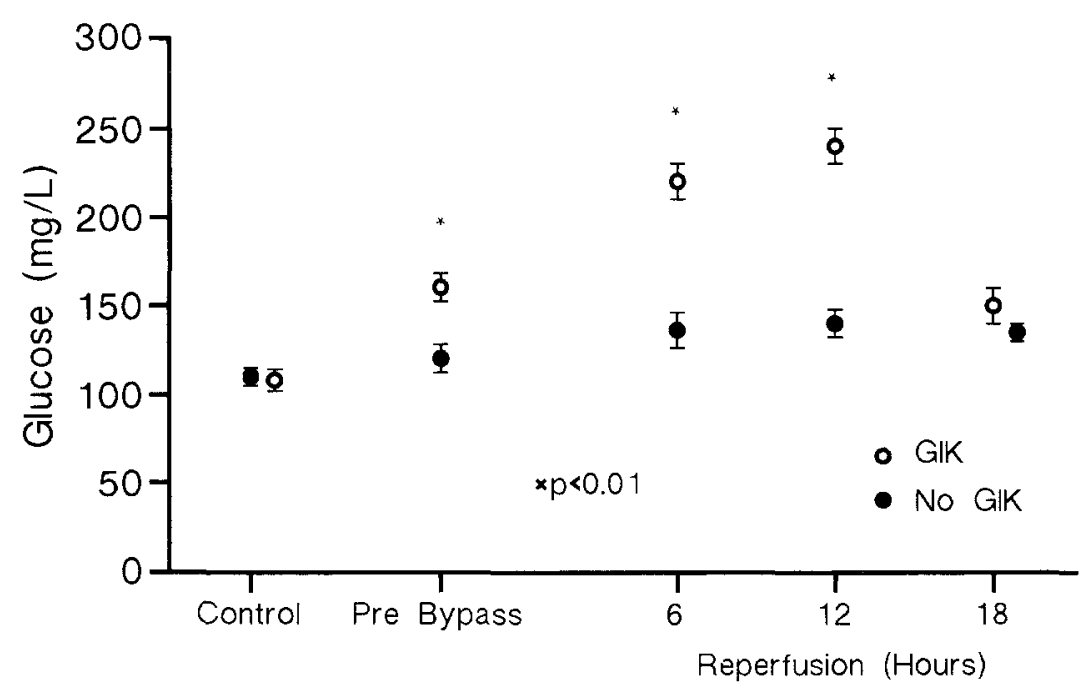

Fig. 2. Serum glucose levels. All values represent the mean plus or minus the standard error. Serum glucose levels are higher in the GIK group during the infusion of GIK solution. After the discontinuation of GIK infusion, serum glucose level is not different from that in the no-GIK group.

This contributed to a shorter length of stay in the ICU $(14.8 \pm 1.3$ hours GIK vs $31.6 \pm 5.2$ hours no-GIK; $p=0.002 ; 95 \%$ CI 5.38 to 28.34 . Patients who received GIK solution also had a lower incidence of atrial fibrillation $(2 / 15$ vs $8 / 15 ; p=$ $0.010)$ and ventricular arrhythmias $(0 / 15$ vs $3 / 15$; $p=0.060)$. The shorter ICU stay and lower incidence of arrhythmia also contributed to a signifcantly shorter hospital stay for patients in the GIK group $(6.0 \pm 0.4$ days GIK vs $8.0 \pm 0.7$ days no-GIK group; $p=0.010 ; 95 \%$ CI 0.29 to 3.83 ).

\section{Discussion}

There are several mechanisms by which GIK solutions enhance the performance of the ischemic myocardium. The increased supply of adenosine triphosphate derived from glycolytic pathways plays an important role in maintaining cell membrane function and integrity, which is critical to the preservation of myocytes and endothelial and vascular smooth muscle cells. ${ }^{14}$ This limits cellular edema and microvascular compression, which contribute to the "no-reflow" phenomenon that may occur during 


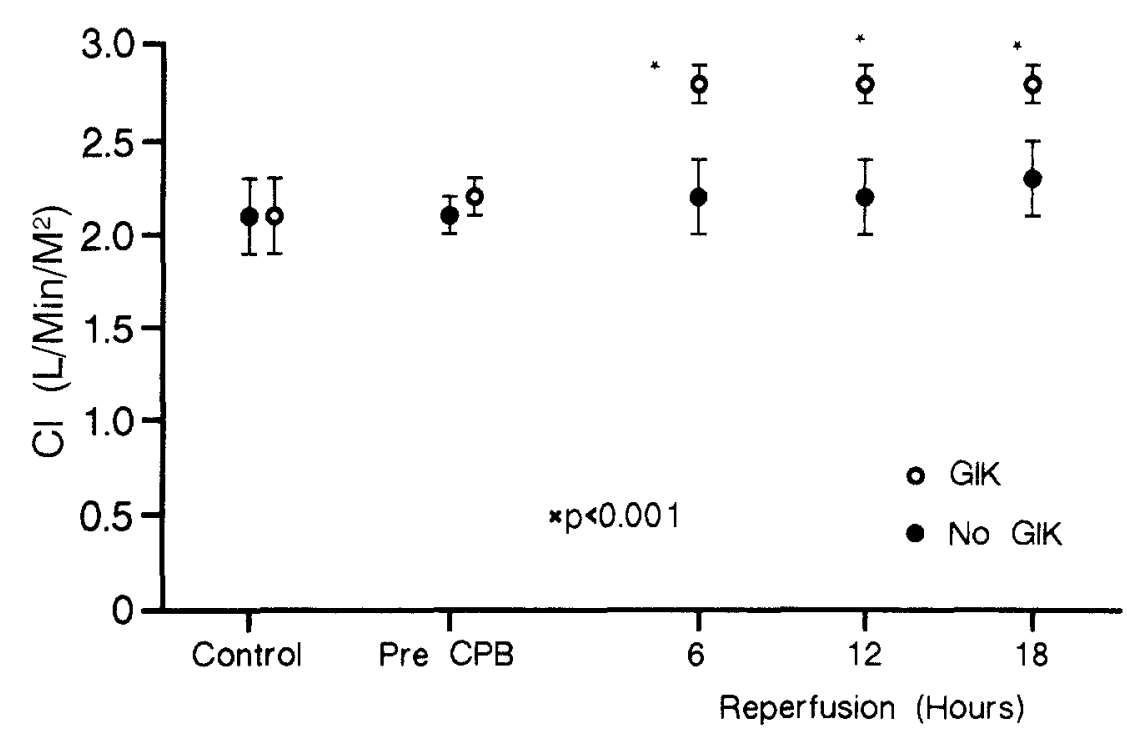

Fig. 3. Cardiac index $(C I)$. All values represent the mean plus or minus the standard error. Cardiac index is significantly higher in the GIK group during reperfusion.

reperfusion. Eberli and coworkers ${ }^{15}$ were able to demonstrate increased blood flow and decreased coronary resistance during reperfusion in ischemic hearts treated with GIK. This decrease in cellular edema may also have contributed to the decreased weight gain seen in the patients in our study who were treated with GIK solution. During ischemia, the accumulation of free fatty acid end products may impair ventricular function and increase the incidence of ventricular arrhythmia by increasing the level of oxygen-derived free radicals. ${ }^{16}$ Glucose esterifies intracellular free fatty acids by increasing the supply of $\alpha$-glycerophosphate, thereby decreasing the toxic metabolic end products of free fatty acids including oxygen-derived free radicals. ${ }^{16,17}$ The beneficial effects of GIK solution on the ischemic myocardium are unlikely to be the result of its hyperosmolarity. In any experimental study with ischemic hypertrophied rat hearts, the substitution of mannitol for glucose failed to show the same degree of myocardial protection. ${ }^{18}$

The superiority of glucose as a substrate in the ischemic myocardium has been documented in several clinical studies. In patients with coronary artery disease, GIK therapy resulted in a more favorable oxygen supply/demand ratio by increasing arterial glucose uptake while decreasing free fatty acid levels. ${ }^{19}$ Similar results were noted by Svedjeholm and colleagues ${ }^{20}$ in patients receiving GIK infusions after elective CABG. Maki and associates ${ }^{3}$ studied the pattern of glucose utilization in patients with an occluded coronary artery and chronic wall motion abnormalities without a previous infarction. Insulin infusions caused a striking increase in glucose uptake in both dysfunctional and normal myocardial regions. These beneficial changes in myocardial metabolism were reflected in improved myocardial performance in patients after anterior myocardial infarcts. ${ }^{7,8}$ Patients treated with GIK infusions showed improved global ejection fraction values and significantly better regional wall motion in the infarcted area. Patients undergoing cardiac operation also appeared to benefit from treatment with GIK. Oldfield, Commerford, and Opie ${ }^{9}$ treated patients with an infused GIK solution for 12 hours before a mitral valve replacement. These patients had a higher myocardial glycogen content during the operation and had a lower incidence of postoperative arrhythmia than patients not treated with GIK. Coleman and coworkers ${ }^{10}$ used GIK infusions in patients who required IABP support to discontinue cardiopulmonary bypass after CABG. Although GIK did not alter mortality or infarction rates, the length of IABP support and the need for inotropic agents were significantly decreased in the patients in the GIK-treated group. Girard and coworkers ${ }^{11}$ noted higher cardiac indices in patients undergoing elective $\mathrm{CABG}$ who received pretreatment with GIK.

One surprising finding in our study was the significant decrease in the incidence of atrial fibrillation in the GIK group. The overall incidence of 
atrial fibrillation in this study was $33 \%$, which is similar to that in our previous series and those of others. ${ }^{21-24}$ Patients treated with GIK had only a $13 \%$ incidence of atrial fibrillation compared with $53 \%$ in patients in the no-GIK group. We and others have shown that postoperative atrial fibrillation increases length of hospital stay and can be associated with an increased incidence of strokes. ${ }^{21,22}$ There are several theories for the increased incidence of atrial arrhythmias after CABG. Kalman and coworkers $^{25}$ showed an increased incidence of higher postoperative norepinephrine levels in patients with atrial fibrillation undergoing CABG. This suggests that increased sympathetic activation may be an important mechanism for the development of atrial fibrillation. There is also evidence to suggest that atrial ischemia during cardioplegic arrest may be an important factor. Tchervenkov and coworkers ${ }^{23}$ noted a strong correlation between the duration of atrial activity during cardioplegic arrest and the incidence of postoperative supraventricular arrhythmia in patients undergoing elective CABG. This suggests that atrial fibrillation may be a manifestation of inadequate atrial protection during myocardial ischemia. Similar findings were also noted by Mullen ${ }^{26}$ and Smith $^{27}$ and their associates who found that the atria in patients undergoing CABG with cold cardioplegic techniques were cooled less effectively than the ventricles. $\mathrm{Cox}^{28}$ suggested that some patients are more vulnerable to postoperative atrial fibrillation because of a mild nonuniformity in the refractory period distribution in the atria of these patients. He believes that atrial ischemia associated with rapid rewarming of the atria during cold cardioplegic arrest is more likely to result in atrial fibrillation in these patients. Pretreatment with GIK solution may increase myocardial glycogen stores and result in less atrial ischemia during cardioplegic arrest. Further evidence to support this theory comes from the work of Oldfield, Commerford, and Opie, ${ }^{9}$ who biopsied the atria in patients who received GIK treatment 12 hours before mitral valve replacement. Patients treated with GIK had higher glycogen levels and a lower incidence of postoperative arrhythmia.

One of the possible limitations of this study is that although the patients were prospectively randomized, the staff caring for the patients knew which patients received GIK treatment. However, because the use of inotropic agents and the decisions to extubate the patients and discharge them from the ICU and hospital were all dictated by established protocols, it is unlikely that bias and selection influenced these results.

GIK therapy was well tolerated and resulted in no adverse reactions. It is cheap, readily available from the hospital pharmacy, and easy to administer. To avoid the possibility of venous trauma caused by hyperosmolarity, GIK solution was only given through a central line. Patients with diabetes were not included in this study but there is new evidence to suggest that mortality in patients with diabetes who have acute myocardial infarctions can be significantly reduced by the infusion of an insulin-glucose solution. ${ }^{29}$ Future studies will be undertaken to determine whether GIK solution will be equally effective in reducing the morbidity and mortality of $\mathrm{CABG}$ in patients with diabetes.

\section{REFERENCES}

1. Jones EL, Weintraub W, Craver J, Guyten RA, Cohen CL. Coronary bypass surgery: is the operation different today? J Thorac Cardiovasc Surg 1991;101:108-15.

2. Opie LH. Effects of regional ischemia on metabolism of glucose and fatty acids: relative rates of aerobic and anaerobic energy production during myocardial infarction and comparison with effects of anoxia. Circ Res 1976;38(suppl 1):52 74.

3. Maki M, Luotolahti $M$, Nuutila $P$, et al. Glucose uptake in the chronically dysfunctional but viable myocardium. Circulation 1996;93:1658-66.

4. Sodi-Pollaris D, Testelli MD, Figleder BL, et al. Effects of an intravenous infusion of a potassium-glucose-insulin solution on the electrocardiographic signs of myocardial infarction. Am J Cardiol 1965;5:166-81.

5. Marako PR, Libby P, Sobel SE, et al. Effect of glucoseinsulin-potassium infusion on myocardial infarction following experimental coronary artery occlusion. Circulation 1972;45: 1160-75

6. Heng MK, Norris RM, Peter T, Nisbet HD, Singh BN. The effects of glucose-insulin-potassium on experimental myocar dial infarction in the dog. Cardiovasc Res 1978;12:429-35.

7. Whitlow PL, Rogers WJ, Smith LR, et al. Enhancement of left ventricular function by glucose-insulin-potassium infusion in acute myocardial infarction. Am J Cardiol 1982;49: 811-20.

8. Satler LF, Green CE, Kent KM, Pallas RS, Pearle DL, Rackley CE. Metabolic support during coronary reperfusion. Am Heart J 1987;114:54-8.

9. Oldfield GS, Commerford PJ, Opie LH. Effects of preoperative glucose-insulin-potassium on myocardial glycogen levels and on complications of mitral valve replacement. J Thorac Cardiovasc Surg 1986;91:874-6.

10. Coleman GM, Gradinac S, Taegtmeyer H, Sweeney M, Frazier $\mathrm{OH}$. Efficacy of metabolic support with glucoseinsulin-potassium for left ventricular pump failure after aortocoronary bypass surgery. Circulation 1989;80(Suppl): I91-6.

11. Girard C, Quentin P, Bouvier H, et al. Glucose and insulin 
supply before cardiopulmonary bypass in cardiac surgery: a double-blind study. Ann Thorac Surg 1991;54:259-63.

12. Gradinac S, Coleman GM, Taegtmeyer H, Sweeney MS, Frazier $\mathrm{OH}$. Improved cardiac function with glucose-insulinpotassium after aortocoronary bypass grafting. Ann Thorac Surg 1989;48:484-9.

13. Lazar HL, Zhang $X$, Rivers $S$, Bernard S, Shemin RJ. Limiting ischemic myocardial damage using glucose-insulinpotassium solutions. Ann Thorac Surg 1995;60:411-6.

14. Owen P, Dennis S, Opie LH. Glucose flux rate regulates onset of ischemic contracture in globally underperfused rat hearts. Circ Res 1990;66:344-54.

15. Eberli FR, Weinberg EO, Grice WN, Horowitz GL, Apstein CS. Protective effect of increased glycolytic substrate against systolic and diastolic dysfunction and increased coronary resistance from prolonged global underperfusion and reperfusion in isolated rabbit hearts perfused with erythrocyte suspensions. Circ Res 1991;68:466-81.

16. Hess ML, Okabe E, Poland J, Warner M, Stewart JR, Greenfield LJ. Glucose-insulin-potassium protection during the course of hypothermic global ischemia and reperfusion: a new proposed mechanism by the scavenging of free radicals. J Cardiovasc Pharmacol 1983;5:35-43.

17. Leidtke AJ. Lipid burden in ischemic myocardium. J Moll Cell Cardiol 1988;20:65-74.

18. Cunningham MJ, Apstein CS, Weinberg EO, Vogel.WM, Lorell BH. Influence of glucose and insulin on the exaggerated diastolic and systolic dysfunction of hypertrophied rat hearts during hypoxia. Circ Res 1990;66:406-15.

19. Stanley AW, Moraski RE, Russell RO, et al. Effects of glucose-insulin-potassium on myocardiol substrate availability and utilization in stable coronary artery disease. Am J Cardiol 1975;16:929-37.

20. Svedjeholm R, Hallhagen S, Ekroth R, Joachimsson PO, Ronquist G. Dopamine and high-dose insulin infusion (glucose-insulin-potassium) after a cardiac operation: effects on myocardial metabolism. Ann Thorac Surg 1991;51:262-70.

21. Lazar HL, Fitzgerald C, Gross S, Heeren T, Aldea GS, Shemin RJ. Determinants of length of stay after coronary artery bypass graft surgery. Circulation 1995;92:II20-4.

22. Creswell LL, Schuessler RB, Rosenbloom M, Cox JR. Hazards of postoperative atrial arrhythmias. Ann Thorac Surg 1993;56:539-49.

23. Tchervenkov CI, Wynands JE, Symes JF, Malcolm ID, Dobell A, Morin JE. Persistent atrial activity during cardioplegic arrest: a possible factor in the etiology of postoperative supraventricular tachyarrhythmias. Ann Thorac Surg 1983;36:437-43.

24. Rubin DA, Nieminski KE, Reed GE, Herman MV. Predictors, prevention, and long term prognosis of atrial fibrillation after coronary artery bypass graft operations. J Thorac Cardiovasc Surg 1987;94:331-5.

25. Kalman J, Munawar M, Howes LG, et al. Atrial fibrillation after coronary artery bypass grafting is associated with sympathetic activation. Ann Thorac Surg 1995;60:1709-15.

26. Mullen JC, Khan N, Weisel RD, et al. Atrial activity during cardioplegia and postoperative arrhythmias. J Thorac Cardiovasc Surg 1987;94:558-65.

27. Smith PK, Burrman WC, Levett J, Ferguson TB Jr, Holman WL, Cox JL. Supraventricular conduction abnormalities following cardiac operations: a complication of inadequate atrial preservation. J Thorac Cardiovase Surg 1983;85:105-15.
28. Cox JL. A perspective of postoperative atrial fibrillation in cardiac operations. Ann Thorac Surg 1993;56:405-9.

29. Malberg K, Ryden L, Efendic S, et al. Randomized trial of insulin-glucose infusion followed by subcutaneous insulin treatment in diabetic patients with acute myocardial infarction (DIGAMI study): effects on mortality at 1 year. J Am Coll Cardiol 1995;26:57-65.

\section{Discussion}

Dr. Steven Guyton (Seattle, Wash.). GIK solutions have been around for quite some time. At the time of my work with George Clowes, the father of Alec Clowes, at the Sears Surgical Laboratory at Boston City Hospital he championed the improved myocardial performance with what he called "GKI" in patients with sepsis and other critically ill patients after operation. His work in substrate enhancement influenced the development of hyperalimentation now commonly used in our surgical ICUs. Perhaps we are now sophisticated enough in our methods of myocardial preservation and evaluation of myocardial performance that improvements with this sort of substrate enhancement will be measurable in clinical situations. I would like to be more clearly convinced.

My greatest concerns with the mechanics of this study are the introduction of bias by the method of randomization, which I presume was done on the basis of even or odd clinic number, and the fact that the study was not blinded. In hindsight in my review of the manuscript it seemed as though doing this as a blinded randomized trial would have been fairly easy to do. In the studies performed, as I understand the randomization protocol, the patients would have been identified with the GIK group or the no-GIK group at the moment of presentation.

The authors state that the patients in the GIK group required less inotropic support, yet GIK solutions themselves enhance myocardial performance. Is GIK included in the inotropic score, or did the authors measure catecholamine levels in the GIK versus no-GIK groups?

Dr. Lazar. Randomization was done by the last digit of the hospital number in those patients identified as being eligible for the study. We found it was difficult to do the study in a blinded fashion because the glucose levels were clearly elevated in patients in the GIK group. Although I share some of Dr. Guyton's concerns, I think this really did not influence the results. It is hard to see how the incidence of atrial fibrillation or ventricular arrhythmia would be affected.

As far as the use of inotropes is concerned, the inotropic use in both groups was relatively small. We did not think of GIK solution as an inotrope. I think that the improvements seen in the cardiac index were the result of improved diastolic relaxation, better stroke work index, and overall general better ventricular performance in GIK-treated hearts.

Dr. Guyton. The authors note a decrease in cellular edema as a potential mechanism for GIK. Because the solution is given systemically, is this lesser weight gain a myocardial phenomenon that we are seeing or is this a systemic effect with regard to the entire body?

Dr. Lazar. This is most likely both a myocardial and systemic effect. 
Dr. Guyton. This is an area of concern with regard to group bias, because the authors noted that there was less weight gain and less edema in the GIK group and that GIK in their evaluation limited fluid retention. Did they measure or record the amounts of fluid administration and urine output during the procedures and during the patient's hospital stay? What can they tell us about the fluid administration and management of both groups?

Dr. Lazar. The fluid administration in both groups was standardized through a standard postoperative protocol, so all these patients were weighed at 5 o'clock in the morning on the first postoperative day and we found as was the case in all our patients that the maximal weight gain was seen on that first postoperative day. We found no variations between the two groups as far as that was concerned.

Dr. Guyton. Was more fluid administered to the GIK group?

Dr. Lazar. Yes, they received more fluid because a faster infusion rate was used in that group.

Dr. Guyton. It would be of interest I think to look at a GIK group and a low-dose inotropically supported or routinely supported group to indicate the inotropic potential of GIK solution. Perhaps now we are on the verge of seeing the marked improvements with substrate enhancement as we saw with hyperalimentation.

Dr. Lazar. There are two reasons we would not do that particular study. First of all, low-dose inotropes are more expensive to use and, more important, what we found from this study is that the incidence of atrial arrhythmia was significantly decreased. At least in our own retrospective series we found that the use of inotropes after operation was associated with a higher incidence of both atrial and ventricular arrhythmia, so I think that GIK is working not as an inotrope and I would think that the use of inotropes would have resulted in a higher incidence of arrhythmia.

Dr. James Albert (Colorado Springs, Colo.). I was wondering whether the authors looked at the differences in urine output during the study period. I am concerned that because the glucose concentrations were significantly higher in the GIK group over a prolonged period, there may be a component of an osmotic diuresis that may be potentially responsible for the decreased weight gain in that group.

Dr. Lazar. There is no question that there was some osmotic diuresis in the GIK group, but I do not think that was responsible for the beneficial results seen in this study. Previous experimental studies in which mannitol was substituted for GIK failed to show the beneficial protective effects on ventricular function.

Dr. Albert. The second question I have is whether the authors were releasing the patients from the ICU in the middle of the night after procedures, because there was a significant difference in the ICU stay. In my experience patients operated on in both the morning and afternoon tend to be released the following morning if all goes well, which would result in a difference in the time in the ICU. Were there any differences in the times of day that the operations were done in both groups?
Dr. Lazar. We did look at that and there were an equal number of afternoon and morning operations in both groups. The criteria for discharge from the ICU and discharge from the hospital are pretty rigid. The patients have to be receiving no inotropic support and be extubated to leave the ICU, and to leave the hospital they have to be afebrile with no active infections and be able to walk at least a certain distance with a certain acceptable oxygen saturation.

Dr. Mark Metzdorff (Portland, Ore.). I am impressed by this carefully done study and intrigued by the salutary effects of this solution, especially as regards atrial fibrillation, because that seems in our experience to be the primary cause of prolonged hospital stay. I think the authors rightly excluded some high-risk patients or some that would potentially confound the findings, such as those with diabetes and chronic renal failure, but in our practice that would account for about $40 \%$ of the patients we operate on. Do the authors anticipate extending this treatment to these patients, or are they absolutely excluded?

Dr. Lazar. Such a protocol is going to be underway shortly with the help of our endocrinologists. I think the bigger issue that we are addressing is that those patients with ischemic heart disease do have an abnormality in the ability to use glucose and an abnormality in the ability to use insulin, and certainly the group that would tend to benefit the most are the patients with diabetes who tend to gain more weight, who have a higher incidence of perioperative infarcts, and in whom it is much more difficult to provide protection during the operation. Recent data in the cardiology literature show that similar GIK-type solutions have dramatically decreased the mortality from acute infarcts in patients with diabetes, and we are actually now modifying the solution to use it in these patients.

Dr. Philip Wright (Honolulu, Hawaii). This article seems to harken back to a period when potassium loading of patients after myocardial infarction was thought to be beneficial in reducing ventricular arrhythmia, as described by Dr. Sodi-Pollaris from Mexico City. I am wondering whether in a simplistic way the authors are telling us that potassium loading in these patients has some benefit inasmuch as I see the serum levels fluctuate very little. Is purely potassium loading independent of any other consideration providing the benefit in these patients?

Dr. Lazar. I would think it is more the glucose than the potassium. I think the key element is getting substrate into the cells, especially in many of these patients who have ischemia before the operation. They are not infarcting, but they have underlying ischemia. I think that surgeons were keying in on that period of ischemic arrest and reperfusion, and we really neglected that period before the patient gets to the operating room.

Dr. Wright. My second question is that there has been some suggestion in a paper presented earlier this week at the DeBakey Society of looking at insulin elaboration in various experimental preparations. There has been a suggestion that during bypass insulin elaboration by the pancreas may be at a reduced level or shut off, in which case adding insulin to this preparation is important. Do the authors have a comment? 
Dr. Lazar. Yes, I agree with that wholeheartedly, and this gets back to the other question that was asked about the use of GIK solution in patients with diabetes. In preparation for the next protocol, we have actually initiated insulin drips in a number of our patients with diabetes rather than trying to give them spot doses of insulin as the glucose levels increase. We have seen a dramatic decrease in the weight gain in these patients, a decrease in the incidence of the use of inotropes, and a little bit faster extubation times. I think the other part of this whole process is the inability of the myocardium to adequately use insulin during the period of cardiopulmonary bypass. It may be that bypass and ischemia act as a depressant and that the other important mechanism involved is the ability of endogenous or exogenous insulin to get more glucose into the cells.

\section{Send us your new address at least six weeks ahead}

Don't miss a single issue of the journal! To ensure prompt service when you change your address, please photocopy and complete the form below.

Please send your change of address notification at least six weeks before your move to ensure continued service. We regret we cannot guarantee replacement of issues missed due to late notification.

\section{JOURNAL TITLE:}

Fill in the title of the journal here.

\section{OLD ADDRESS:}

Affix the address label from a recent issue of the journal here.

\section{NEW ADDRESS:}

Clearly print your new address here.

Name

Address

City/State/ZIP
COPY AND MAIL THIS FORM TO:

Journal Subscription Services

Mosby-Year Book, Inc.

11830 Westline Industrial Dr.

St. Louis, MO 63146-3318
OR FAX TO:

314-432-1158

Mosby
OR PHONE:

1-800-453-4351

Outside the U.S., call

314-453-4351 